\title{
Positive Expiratory Pressure Oxygen Therapy (PEP- OT): Innovation in acute respiratory support
}

\author{
Authors: Nitin Dhochak ${ }^{1}$, Rakesh Lodha ${ }^{1}$, Sushil K Kabra ${ }^{1}$ \\ ${ }^{1}$ Department of Pediatrics, All India Institute of Medical Sciences, New Delhi, India
}

\section{Correspondence to:}

Dr Nitin Dhochak, Research Officer, Department of Pediatrics, AIIMS, Ansari Nagar, Delhi, India - 110029. E-mail ID: nitind.aiims@gmail.com

Conflict of Interest: None

Funding source: None

\begin{abstract}
Respiratory support is commonly required in emergency department and hospitalized patients. Standard oxygen therapy through nasal prong or face mask administers high oxygen concentration, but does not have any effect on physiological alterations of alveolar collapse and work of breathing. High flow nasal canula (HFNC) and continuous positive airway pressure (CPAP) devices offer mechanical benefits with improvement in functional residual capacity along with oxygen therapy, but need expensive equipment. We developed a novel oxygen delivery device which combines beneficial effects of positive expiratory pressure with oxygen therapy. Positive expiratory pressure oxygen therapy (PEP-OT) device is prepared by using commercially available positive end-expiratory pressure (PEEP) valve, airtight face mask and oxygen reservoir from a non-rebreathing mask attached via a T-/Yconnector. Typically, air-flow should be slightly more than minute volume and initial PEEP is set at 5-7 cm of water. PEP-OT offers a low-cost, light-weight device which can be useful even in remote areas where electricity might not be available. It could potentially be an alternative to HFNC and CPAP therapy in patients requiring acute respiratory support. With unprecedent demand of respiratory equipment in COVID-19 pandemic, PEP-OT could be an attractive alternative respiratory support.
\end{abstract}




\section{Background}

Oxygen support is frequently administered to patients during pre-hospital transport, emergency management, as well as hospital stay. Traditional respiratory supports include standard oxygen therapy (SOT) (nasal prong, face mask, and non-rebreathing masks), high flow nasal canula (HFNC), non-invasive support [continuous positive airway pressure (CPAP), and bilevel positive airway pressure (BIPAP)] and invasive mechanical ventilation (1). Most important mechanisms of hypoxemia in acutely ill patients are impaired diffusion of gases due to thickened alveolocapillary membrane (interstitial infiltrates and edema seen in early phases of pneumonia and pulmonary edema), and shunting due to complete nonparticipation of affected alveoli which are flooded with secretions or collapsed (dense consolidation and collapse). Apart from hypoxemia, these changes also lead to impaired compliance and hence, increased work of breathing. Persistently increased work of breathing leads to highly negative intrapleural pressure, which can further aggravate the established lung injury by lung strain, also known as patient self-inflicted lung injury (P-SILI) $(2,3)$.

SOT provides high oxygen concentration in inspired gases, hence improving blood oxygen concentration and hemoglobin saturation by overcoming diffusion defects. But SOT doesn't have any major effects on pathophysiological changes of collapsed alveoli and hence, the work of breathing and P-SILI (1). Respiratory supports which provide end-expiratory pressure (HFNC, CPAP and BIPAP), improve functional residual capacity (FRC) by preventing alveolar collapse during expiration, leading to better lung compliance and less work of breathing. A recent meta-analysis demonstrated improved survival in patients with face-mask and helmet NIV compared to SOT in adults with acute respiratory failure (4). But all these therapies require special equipment or ventilators, which are expensive. These equipment mostly run on electricity (with some power back up), limiting their use during transport and in remote areas. These factors limit availability and utilization of such equipment in economically weaker economies, especially in remote hospitals. Unprecedent need for respiratory support devices during COVID-19 pandemic has further strained the demand-supply balance for these equipment (5). We, hereby, describe assembly, physiological basis, and potential use of a novel low-cost respiratory support device, positive expiratory pressure-oxygen therapy (PEP-OT).

\section{Assembly}

Schematics of PEP-OT device are shown in Figure 1. The assembly consists of a positive end-expiratory pressure (PEEP) valve, an air-tight face mask, and a reservoir from nonrebreathing mask attached via a T- or Y-shaped connector. During inspiration, gases flow from reservoir and gas inlet to the patient and one-way PEEP valve prevents air to enter from surrounding environment. During expiration, patient exhales gases against an adjustable positive pressure through the PEEP valve, while one-way valve prevents expiratory gases to enter the reservoir. The reservoir is filled with fresh gases during expiration. 


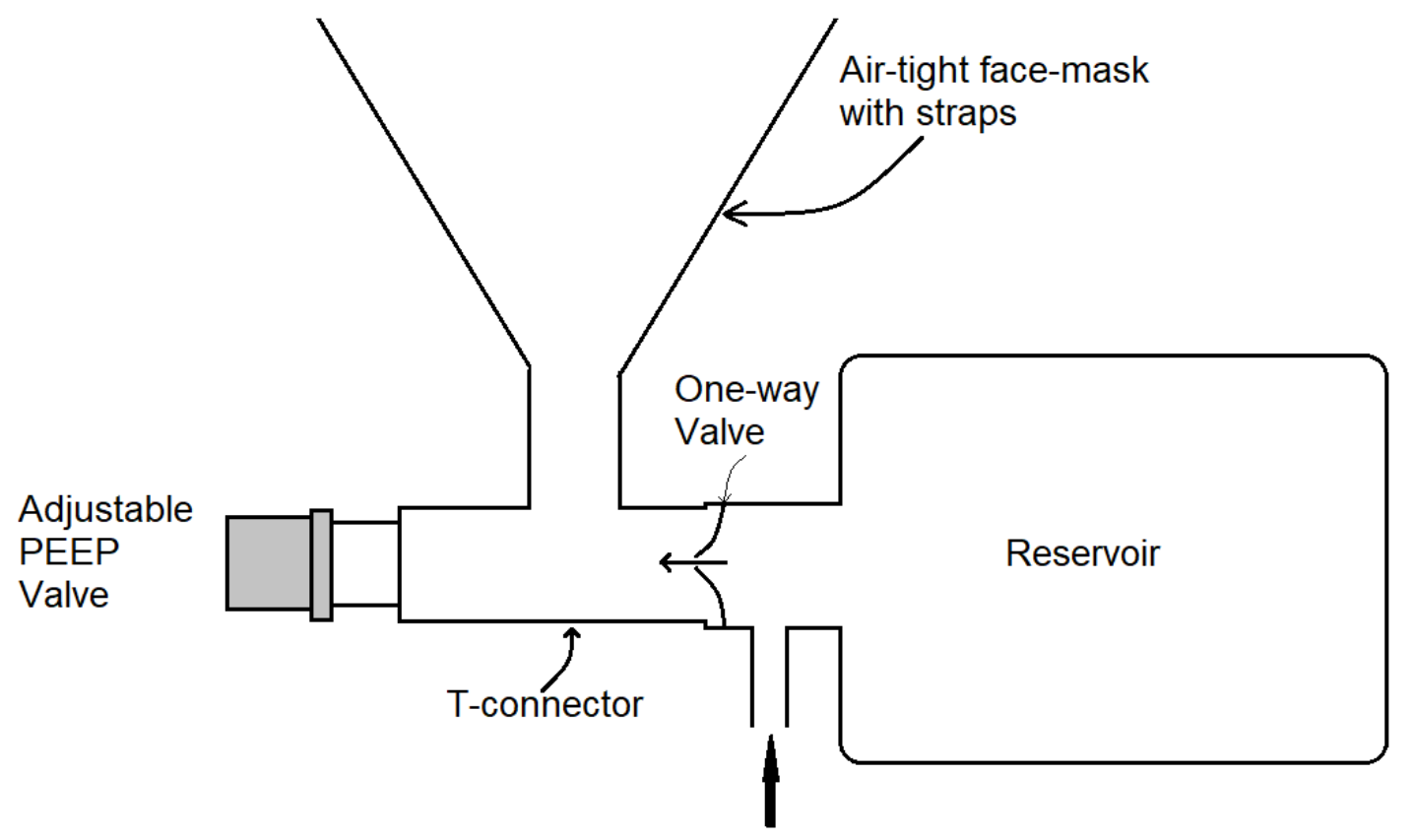

Gas in-flow

Figure 1. Schematic assembly of PEP-OT.

\section{Physiological basis}

PEP-OT is based on principle of positive expiratory pressure (PEP) therapy which is an established chest physiotherapy technique, commonly used in patients with bronchiectasis, lung collapse, and prevention of lung atelectasis in post-operative patients (following heart and chest surgeries) (6). PEP therapy works by distending airways and preventing collapse of alveoli during expiration, and hence improving FRC. PEP therapy has been shown to improve FRC and tidal volume in healthy as well as children with cystic fibrosis (7). PEP therapy in form of expiratory positive airway pressure (EPAP) devices has also been found effective in obstructive sleep apnea, where it is proposed to work by splinting of airway, improved FRC and mild $\mathrm{CO}_{2}$ retention leading to improvement in respiratory drive $(8,9)$.

PEP-OT combines these beneficial effects of PEP on lung physiology with oxygen delivery. The device provides un-interrupted flow of inspiratory gases from the reservoir. The physiological effects are likely to be similar to CPAP therapy. In lung parenchymal pathologies (e.g. pneumonia, pulmonary edema), positive expiratory pressure will improve FRC and decrease work of breathing by preventing collapse of alveoli during expiration. This should also decrease P-SILI and need for advanced respiratory support. In airway diseases [e.g. chronic pulmonary obstructive disease (COPD), bronchiectasis], PEP will help in splinting of airways and decrease auto-PEEP (10). 


\section{Components}

\section{PEEP valve}

PEEP valves are commercially available spring-action based one-way valves. These valves are commercially available to deliver a PEEP range of $0-10 \mathrm{~cm}$ of water (up to $20 \mathrm{~cm}$ of water for few devices) (Figure 2) (11). PEEP can be adjusted by adjusting the tension in spring with rotation of the cap on the device. In PEP-OT, PEEP-valve provides positive pressure during expiratory phase of respiration.

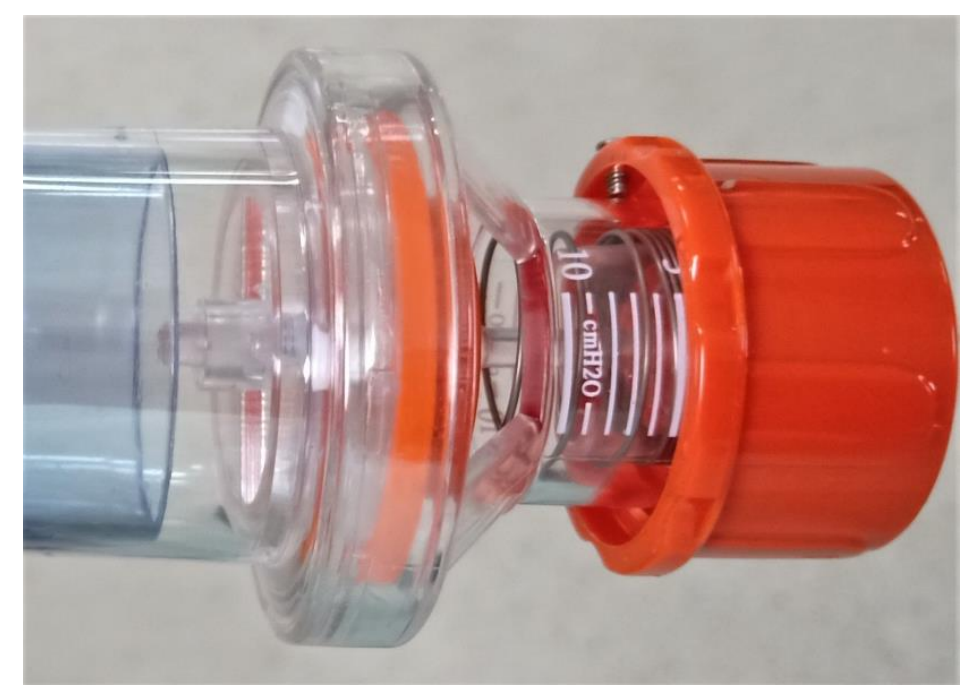

Figure 2. Commercially available PEEP-valve. Orange coloured knob can be rotated to adjust PEEP.

\section{Air-tight face mask}

Non-vented face mask interface with straps is used to deliver PEP-OT while preventing loss of pressure. Non-vented air-cushioned anaesthesia mask or oronasal non-invasive ventilation (NIV) mask can be utilized for the same.

\section{Reservoir}

Reservoir acts as storage for oxygen/gases during expiration and supply uninterrupted gas with high oxygen concentration during inspiration. Reservoir is connected to T- or Yconnector assembly by a one-way valve which prevents entry of exhaled gases to reservoir. Reservoir bag and valve assembly from a commercially available non-rebreathing valve can be used (Figure 3). Reservoir bag should have sufficient volume that it doesn't completely empty during inspiration. Flow should be adjusted such that reservoir bag doesn't empty completely during inspiration. Use of reservoir bag assembly makes a closed system and can delivery nearly $100 \%$ oxygen, but requires high oxygen flow. If a corrugated tube open-end reservoir with one-way valve is used, it can operate at lower oxygen flow, and provide lower 
oxygen concentration. Oxygen concentration can be modified by using blender, especially inhospital.

Potential uses of the PEP-OT device are:

1. As an alternative to SOT for patients with respiratory distress in emergency, ward, pre-hospital and in-hospital transport. PEP-OT could be more effective alternative to SOT and could potentially decrease need for advanced respiratory support as seen with CPAP therapy in certain conditions $(12,13)$.

2. As low cost and easy to use alternative to HFNC or CPAP for patients failing SOT or persistent distress on SOT in emergency department as well as in-hospital.

\section{Indications}

PEP-OT could be used in variety of diseases (where NIV/CPAP is used) including parenchymal pathologies (pneumonia, aspiration, pulmonary edema) as well as airway diseases (bronchiectasis, COPD, acute asthma exacerbation). PEP-OT could be beneficial in patients with congestive heart failure (CHF) by improving work of breathing as well as decreasing preload (decreasing venous return) and afterload (decreasing transmural ventricular load) (14).

PEP-OT would not be used in patients with chronic $\mathrm{CO}_{2}$ retention (while using $100 \%$ oxygen), hemodynamic instability, moderate and severe acute respiratory distress syndrome (ARDS), altered sensorium, poor respiratory efforts, recurrent vomiting, and upper gastrointestinal bleeding (15).

\section{Procedure}

PEP-OT assembly is prepared and attached to humidified oxygen source at high flow (starting at $12-15 \mathrm{~L} / \mathrm{min}$ for adults) (Figure 3 ). For patient with primarily parenchymal defects, initial PEP is set at 5-7 cm of water which can be titrated up in steps of 2-3 cm of water by monitoring patient comfort and hemodynamic stability. For airway diseases, PEP can be set at $4-5 \mathrm{~cm}$ of water (1). The oxygen flow can be titrated such that reservoir bag is not completely empty during any point of respiratory cycle (flow in $\mathrm{L} / \mathrm{min}$ just above the minute ventilation volume). Typically, $15-20 \mathrm{~L} / \mathrm{min}$ oxygen flow will be required for adult patients with respiratory distress. If using open-end corrugated tube reservoir, lower flow rates $(8-12 \mathrm{~L} / \mathrm{min}$ ) could be used. Delivered PEP can be confirmed using a pressure gauze by attaching a pressure line to NIV face mask. Patients on PEP-OT should be monitored for respiratory (respiratory rate, $\mathrm{SpO}_{2}$, work of breathing) and hemodynamic status (heart rate, blood pressure). PEP-OT should be discontinued if respiratory distress is not improving (improvement in respiratory rate and retractions), new onset or worsening of hemodynamic instability or patient having significant discomfort. 


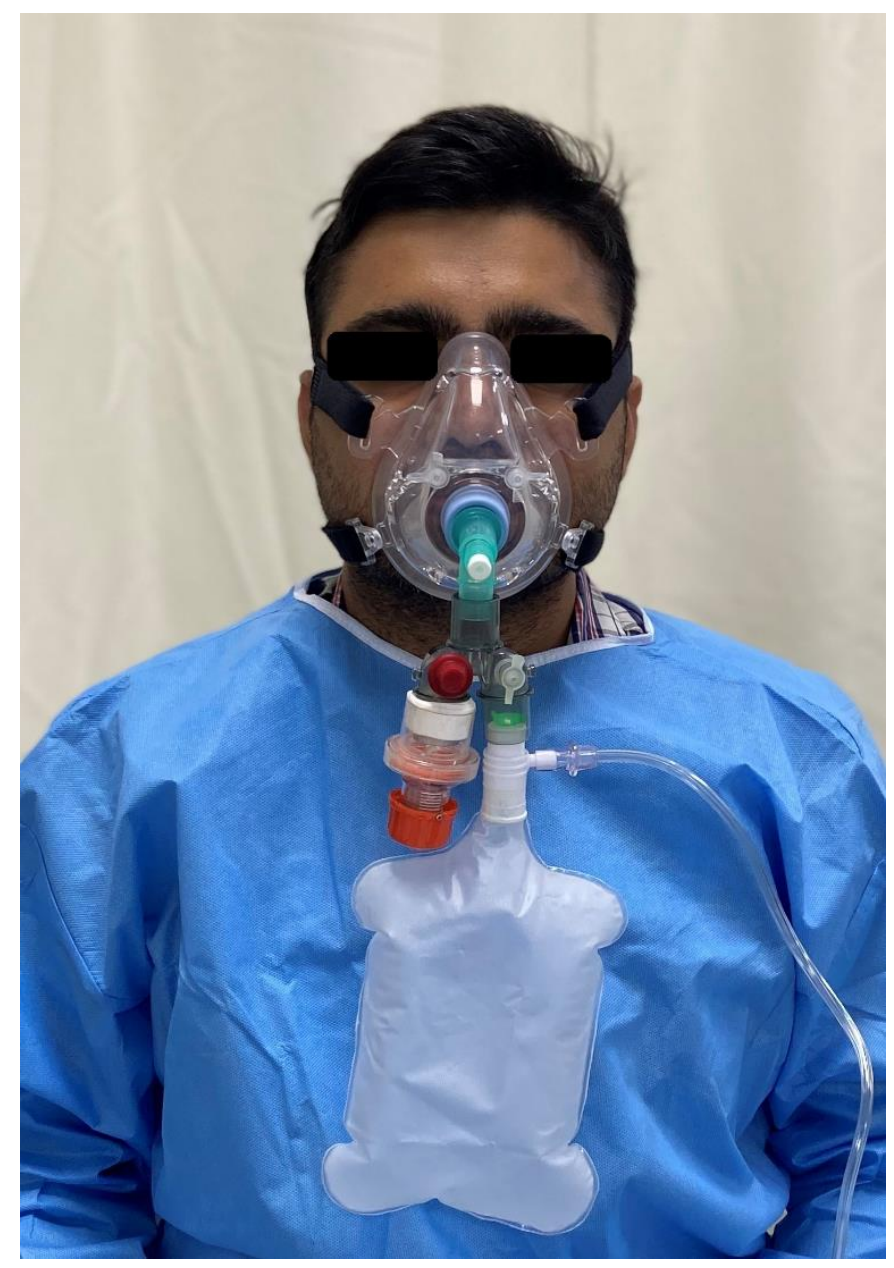

Figure 3. Assembly of PEP-OT. During inspiration, reservoir collapses partially as oxygen flows to the patient.

\section{Cost of therapy}

Cost of PEP-OT device per patient will include cost of non-rebreathing mask for reservoirvalve assembly (\$5 approx.), and PEEP-valve (\$10-15 approx., which can be decreased to approximately $\$ 2 /$ patient as PEEP-valves can be re-used). Non-vented air-cushioned anaesthesia mask or NIV oronasal mask and straps are commonly available in hospitals and can be re-used as many times as possible. If compared to traditional SOT device (nonrebreathing mask) for transport and emergency oxygen support, PEP-OT is likely to cost approximately \$2-3 extra per patient. Overall cost of therapy will need to be adjusted with potentially less need for add-on support with PEP-OT compared to SOT. PEP-OT also doesn't need special expensive equipment such as HFNC machine, NIV machine or ventilator.

Current design is based on redirecting use of already available equipment. Bulk production can be done with more re-usable design, which will decrease the overall cost per patients drastically. 


\section{Advantages of PEP-OT}

1. Doesn't need electricity for operation, hence better applicability in remote areas.

2. Light weight device can be easily used while transporting patients (pre-hospital as well as in-hospital).

3. Can be used with oxygen cylinder (doesn't need high pressure wall oxygen source).

4. Low cost.

5. Not dependent on expensive/special equipment. It could potentially fulfil unmet oxygen therapy device needs during COVID-19 crisis.

6. PEP-OT is free of asynchrony which can be very severe in some devices delivering CPAP or NIV (16). PEP-OT could have advantage over CPAP or NIV by preventing asynchrony related lung injury.

Some commercial devices have been devised to deliver CPAP using mask with CPAP valve and oxygen but these devices do not produce stable pressure. As the resistance of CPAP valve in these devices varies with air-flow rate and turbulence, these were associated with significant decrease in pressure during inspiration and significantly high pressure during expiration, especially at high respiratory drive seen in acutely ill patients (17). As the PEEPvalve is a pop-off valve with fixed pressure and resistance, expiratory pressure does not go beyond set pressure with use of PEP-OT. Presence of reservoir prevents flow starvation and formation of any significant negative pressure formation during inspiration.

\section{Concerns}

As with any positive airway pressure device, PEP-OT could potentially worsen hemodynamic stability by reducing venous return (18). But lack of positive pressure during inspiration is likely to attenuate these adverse hemodynamic effects with PEP-OT. Disconnections in assembly could lead to air leakage. Inadequate flow or increase in respiratory efforts could lead to emptying of oxygen reservoir and air hunger. This can be prevented by using PEP-OT in settings where monitoring is readily available for trouble shooting. Patients with no significant improvement with PEP-OT, should be started on other respiratory support devices including HFNC and CPAP.

\section{Research}

We are planning studies to assess tolerability and any adverse events of PEP-OT in patients with respiratory distress. Once feasibility is established, we will compare PEP-OT as an alternative superior to SOT, and a non-inferior alternative to HFNC or CPAP therapy in patients with respiratory distress.

To conclude, PEP-OT is an attractive and physiologically favourable oxygen delivery alternative with wide range of potential applications. PEP-OT could be even more important in current scenario with unprecedent burden on oxygen therapy devices due to COVID-19 pandemic. 


\section{References}

1. Scala R, Pisani L. Noninvasive ventilation in acute respiratory failure: which recipe for success? Eur Respir Rev. 2018;27: 180029.

2. Grieco DL, Menga LS, Eleuteri D, Antonelli M. Patient self-inflicted lung injury: implications for acute hypoxemic respiratory failure and ARDS patients on non-invasive support. Minerva Anestesiol. 2019;85:1014-23.

3. Yoshida T, Grieco DL, Brochard L, Fujino Y. Patient self-inflicted lung injury and positive end-expiratory pressure for safe spontaneous breathing. Curr Opin Crit Care. 2020;26:59-65.

4. Ferreyro BL, Angriman F, Munshi L, Sorbo LD, Ferguson ND, Rochwerg B, et al. Association of Noninvasive Oxygenation Strategies With All-Cause Mortality in Adults With Acute Hypoxemic Respiratory Failure: A Systematic Review and Meta-analysis. JAMA. 2020;324:57-67.

5. Beitler JR, Mittel AM, Kallet R, Kacmarek R, Hess D, Branson R, et al. Ventilator Sharing during an Acute Shortage Caused by the COVID-19 Pandemic. Am J Respir Crit Care Med. 2020;202:600-4.

6. Fagevik Olsén M, Lannefors L, Westerdahl E. Positive expiratory pressure - Common clinical applications and physiological effects. Respiratory Medicine. 2015;109:297-307.

7. Brilhante SA, Florêncio RB, Gualdi LP, Resqueti VR, Aliverti A, Andrade A de FD, et al. Comparison of different levels of positive expiratory pressure on chest wall volumes in healthy children and patients with fibrosis. Fisioterapia e Pesquisa. 2017;24:311-20.

8. Riaz M, Certal V, Nigam G, Abdullatif J, Zaghi S, Kushida CA, et al. Nasal Expiratory Positive Airway Pressure Devices (Provent) for OSA: A Systematic Review and MetaAnalysis. Sleep Disord. 2015;2015:734798.

9. Berry RB, Kryger MH, Massie CA. A novel nasal expiratory positive airway pressure (EPAP) device for the treatment of obstructive sleep apnea: a randomized controlled trial. Sleep. 2011;34:479-85.

10. Natalini G, Tuzzo D, Rosano A, Testa M, Grazioli M, Pennestrì V, et al. Effect of external PEEP in patients under controlled mechanical ventilation with an auto-PEEP of $5 \mathrm{cmH} 2 \mathrm{O}$ or higher. Ann Intensive Care. 2016;6:53.

11. Ambu® PEEP Valves - Disposable and Reusable. Available from: https://www.ambu.com/emergency-care-and-training/resuscitators/product/peep-valves. Accessed $25^{\text {th }}$ September 2020

12. Gray A, Goodacre S, Newby DE, Masson M, Sampson F, Nicholl J. Noninvasive Ventilation in Acute Cardiogenic Pulmonary Edema. N Engl J Med. 2008;359:142-51. 
13. Delclaux C, L'Her E, Alberti C, Mancebo J, Abroug F, Conti G, et al. Treatment of acute hypoxemic nonhypercapnic respiratory insufficiency with continuous positive airway pressure delivered by a face mask: A randomized controlled trial. JAMA. 2000;284:2352-60.

14. Köhnlein T. CPAP and/or NIV in cardiogenic pulmonary oedema. Breathe. 2009;6:45-9.

15. Nava S, Hill N. Non-invasive ventilation in acute respiratory failure. The Lancet. 2009;374:250-9.

16. Vignaux L, Vargas F, Roeseler J, Tassaux D, Thille AW, Kossowsky MP, et al. Patientventilator asynchrony during non-invasive ventilation for acute respiratory failure: a multicenter study. Intensive Care Med. 2009;35:840-6.

17. Sehlin M, Törnell SS, Öhberg F, Johansson G, Winsö O. Pneumatic performance of the Boussignac CPAP system in healthy humans. Respir Care. 2011;56:818-26.

18. Fessler HE. Effects of CPAP on venous return. J Sleep Res. 1995;4:44-9. 